Pensamiento Crítico Vol. 20 N 1, pp. 75-96

\title{
Determinantes de la violencia urbana en Lima Metropolitana y Callao: Casos de delitos contra el patrimonio*
}

Determinants of urban violence in Lima Metropolitana and Callao: Crimes against property cases

[Recepción: Marzo de 2015/ Conformidad: Mayo 2015]

$$
\text { Juan León Mendoza** }
$$

\section{RESUMEN}

El artículo tiene como objetivo fundamental especificar las variables que coadyuvan a la generación de los delitos contra el patrimonio (robos y asaltos).

Actualmente, el delito contra el patrimonio es el problema de violencia e inseguridad urbana más importante en la capital peruana e inclusive su grado de incidencia tiende a incrementarse.

* Versión resumida de la investigación efectuada en el marco del Programa de Proyectos Multidisciplinarios de Investigación 2014, financiado por el Vicerrectorado de Investigación de la UNMSM. Colaboraron en la ejecución de la investigación: Miguel Inga Arias, Pablo Rivas Santos, Alberto Vásquez Tasayco, Ciro Calero Castillo, Cornelio Ticse Nuñez, Blanca Reyna Marín, Renzo Guzmán Anaya y Giovanni Villalobos Beuzeville.

** Magister en Economía, docente Principal de la Facultad de Ciencias Económicas de la UNMSM. Correo: jleonm@unmsm.edu.pe. 


\section{Pensamiento Crítico Vol. 20. NoI}

Según los resultados de las regresiones econométricas efectuadas, los principales determinantes de la generación de los delitos contra el patrimonio son: la desigualdad en la distribución de ingresos, el consumo de droga, la densidad poblacional y la pobreza; siendo las elasticidades estimadas de $4.23,0.20,0.17$ y -0.15 respectivamente.

Palabras Clave: violencia urbana, inseguridad urbana, robo, asalto

Clasificación JEL: K11

\section{ABSTRACT}

The article's main purpose is to specify the variables that contribute to the generation of crimes against property (robberies and assaults).

Currently, crime against property is the most important problem of violence and urban insecurity in the Peruvian capital and the degree of incidence tends to continue to increase.

According to the results of the regressions performed, the main determinants of the generation of these crimes are: inequality in income distribution, drug use, population density and poverty. The elasticities are estimated to be $4.23,0.20$, 0.17 and -0.15 respectively.

Keywords: Urban violence, urban insecurity, robbery, felony, assault. 


\section{Introducción}

Actualmente, la delincuencia y la inseguridad representa el problema más importante para la población de Lima Metropolitana con un porcentaje de $82 \%$, el cual refleja una tendencia a incrementarse, debido a que en el año 2010, la tasa era de $73 \%$ (Lima Cómo Vamos, 2015).

Por otro lado, a nivel del Perú, el delito contra el patrimonio representa el delito más importante con un $66.85 \%$ del total de delitos. En tanto que a nivel de Lima Metropolitana dicho tipo de delito significa el 73\% del total (INEI, 2013).

Esta información indica con claridad que el problema de la delincuencia y la inseguridad es mayor en Lima y Callao que en cualquier otra región o ciudad del Perú.

En el marco señalado, en este artículo trataremos de especificar las principales variables que coadyuvan a la generación de los delitos contra el patrimonio, mediante la formulación de un modelo microeconómico y la verificación empírica correspondiente utilizando la técnica econométrica de los mínimos cuadrados ordinarios.

\section{Marco teórico y antecedentes}

El concepto de violencia es bastante amplio y difuso, por lo que existen dificultades para definirlo con precisión, pues involucra una gran cantidad de campos interdisciplinarios, además que se refieren a un conjunto de hechos muy heterogéneos que parecieran no tener relación entre sí (Davis, 1987).

Referido a la violencia urbana, inclusive el término "urbano" difiere entre países y ciudades. Por ello, dado que no existe una sola definición del concepto de violencia urbana -salvo de que se desarrolla en la ciudad-, se tiende a describir en función de cinco variables: características directas e indirectas, intensidad y duración, características espaciales y sociales, intencionalidad y contexto. También se suele aproximar la definición de violencia urbana a los actos criminales que se producen en las ciudades en el contexto de las relaciones sociales entre los individuos, cuyas manifestaciones más importantes son: los robos a mano armada, las agresiones, los homicidios, secuestros (Vanderschueren, 1994). En el mismo sentido, la violencia urbana se asocia a actos 


\section{Pensamiento Crítico Vol. 20. NoI}

criminales que generan lesiones o daños físicos a las personas y que por ende, también se manifiesta en la forma de atracos, robos, tentativas de homicidios, violación, violencia familiar, lesiones de tránsito terrestre, homicidios (Del Olmo, 2000).

¿Cúales son las causas o factores que contribuyen a la generación de la violencia urbana?

En principio, la violencia urbana se conceptúa como un fenómeno multicausal, en el que concurren factores individuales, familiares, sociales y culturales (BID, 1998). En este contexto, dada la complejidad de su definición, no sorprende encontrar diversos puntos de vista téoricos y empíricos.

Briceño-León (2007), en una línea sociológica, clasifica las causas y los factores que generan la violencia urbana en tres niveles: (a) macrosociales (la desigualdad social debido al incremento de la riqueza y la pobreza, la paradoja del mayor nivel educativo de las personas, las menores oportunidades de empleo, el incremento en las expectativas y la imposibilidad de satisfacerlas); (b) mesosociales (el incremento de la densidad en las zonas pobres y la segregación urbana, la cultura de la masculinidad); y (c) microsociales (el incremento de las armas de fuego, el consumo de alcohol).

La violencia es también una consecuencia del rápido proceso de crecimiento y modernización de las zonas urbanas. Este hecho altera rápidamente las relaciones sociales y económicas, generando sectores o zonas urbanas marginales en los que se trastoca la escala de valores y la moral de la población. En este marco, la violencia y la delicuencia aparece como un mecanismo de autodefensa de los desplazados, los mismos que se traducen en robos, hurtos, asaltos, lavado de dinero, fraude electrónico (Moulian, 1997). En este mismo sentido, Briceño-León (2002) hipotiza que la violencia urbana se genera por la desigualdad y el proceso de empobrecimiento de las zonas urbano marginales.

Según Brender (2012), las diversas teorías existentes sobre la violencia en general se pueden clasificar en cinco tipos: desorganización social, las ventanas rotas, modelo ecológico, capital social y cohesión social, empoderamiento y empleo de la juventud. Según el primer enfoque, la desorganización social, como consecuencia de la heterogeneidad étnica y las desventajas económicas, se traducen en violencia. Para 


\section{Juan León Mendoza}

el enfoque de las ventanas rotas, la violencia es generada por el desorden urbano. La teoría del capital social y cohesión social postula que la falta de confianza interpersonal y las relaciones sociales desvinculadas producen actos de violencia. La corriente del empoderamiento y empleo de la juventud sostiene que la falta de oportunidades sociales y económicas para los jóvenes ocasiona comportamientos violentos o antisociales de éstos.

Dada la asociación de la violencia urbana con la delincuencia, todo el análisis teórico de la delincuencia, desde el punto de vista de la ciencia económica, se basa en el trabajo de Becker (1968). La teoría económica asume que la persona que comete delito es un agente racional que maximiza utilidad o bienestar. Cuando éste comete delito compara el beneficio neto de dedicarse a la actividad ilegal (delitos) con el beneficio neto que le reporta la actividad legal. Si el beneficio de la actividad ilegal es mayor que el de la legal, entonces el individuo delinque. A su vez, el beneficio neto de la actividad ilegal o delictiva se define como el ingreso que genera dicha actividad deducidos los costos en que se incurre en ésta. Si el ingreso es mayor que el costo, entonces el beneficio neto de la actividad delictiva será positiva por lo que habrá incentivo para cometer delitos.

En la corriente economicista de la violencia, Del Olmo (2000) sostiene, que los robos y atracos, que pertenecen a hechos de violencia, tienen motivación y dimensión económica. Sin embargo, lo económico va más allá de los robos y atracos, en la medida en que estos hechos están en el terreno de la vigencia del estado de derecho y la defensa de la propiedad, que también forman parte de la ciencia económica.

La United Nations Office on Drugs and Crime (UNODC, 2008), en su informe del año 2008 clasifica los factores de riesgo generador de la criminalidad en tres campos diferentes: nivel macro (beneficio esperado neto y coste de oportunidad de delinquir, la restricción moral); nivel micro (desigualdad en el ingreso per cápita, mala calidad de las instituciones, sistema punitivo laxo, fácil acceso a las armas, alto desempleo, volatilidad económica, baja demanda de los trabajadores calificados, baja calidad educativa); y nivel individual (círculo de amistades, consumo de droga, capacidades cognitivas o IQ bajo, posesión de características objeto de discriminación, falta de motivación, egoísmo, bajo nivel educativo de los padres, antecedentes criminales en la familia, poca disciplina en la educación, abuso de droga de los padres, fracaso escolar y discriminación en la escuela). 


\section{Pensamiento Crítico Vol. 20. NoI}

Según Brender (2012), entre las principales variables que las diferentes investigaciones empíricas señalan para explicar la violencia urbana destacan: la rápida urbanización y crecimiento de la población, la densidad poblacional urbana, la pobreza urbana y desigualdad, la juventud urbana y desempleo y, las fallas de gobernanza urbana.

En el marco de lo señalado por Brender, la rápida urbanización y crecimiento de la población ha exacerbado la pobreza y la degradación ambiental, lo que ha generado problemas de violencia (Martine y McGranahan, 2010). Sin embargo, los resultados sobre la relación entre la densidad de la población y la violencia no son necesariamente en sentido directo (Hasan 2010). También se destaca que la desigualdad en la distribución de ingresos tiene una mayor fuerza explicativa que la pobreza en la determinación de los actos criminales, en tanto que el nivel del ingreso per cápita no tiene relevancia explicativa. Según el trabajo de Jutersonke et al. (2009), el incremento demográfico de la población juvenil asociada a la formación de pandillas, explica la generación de la violencia organizada. Por otro lado, la incapacidad de los gobiernos para controlar la ciudad y hacer cumplir las reglas urbanas, promueve el surgimiento de grupos violentistas (Townsend 2009).

Stewart (2008) encuentra que la violencia es mayor en las zonas urbanas de menores niveles de ingreso, las mismas que usualmente son altamente concentradas y más pobres. En éstas las tasas de homicidios son mayores que el promedio. Así la violencia golpea más a los pobres y se genera un círculo vicioso entre la violencia, desigualdad en la distribución de ingresos y la pobreza.

Según el estudio efectuado por Winton (2004), el tráfico de drogas es también otra de las variables que coadyuva a la violencia urbana. La actividad del narcotráfico no sólo genera corrupción, sino también control sobre zonas marginales donde impone su ley, recurriendo si es necesario a medidas de violencia extrema. Además, las organizaciones dedicadas al narcotráfico muchas veces ejecutan actividades comunitarias en estas zonas marginales, creando así lealtades y simpatías, lo cual tiende a institucionalizar la falta de autoridad gubernamental y el estado de derecho.

En el ámbito internacional, entre los trabajos relevantes de corte econométrico, destaca el efectuado por Fajnzylber et al. (1998). Estos autores regresionan la 


\section{Juan León Mendoza}

delincuencia con el producto nacional bruto percápita, el índice de desigualdad Gini, la educación media, la tasa de urbanización y el consumo de drogas. Encuentran que sólo el índice Gini explica de manera sistemática la ocurrencia de la violencia en los países latinoamericanos.

\section{El modelo}

Antes de especificar econométricamente los factores que generan la violencia urbana o la comisión de delitos contra el patrimonio, en la línea microeconómica del premio Nobel Gary Becker (1968), en este capítulo formularemos un modelo teórico que permitirá ilustrar y explicar teóricamente la generación del citado problema.

Definiremos al individuo que comete el delito como un delincuente. El delincuente, en el tiempo, es un individuo racional que trata de alcanzar el máximo nivel de bienestar o utilidad familiar (U) en el tiempo. La utilidad individual (u) multiplicada con el número de miembros que conforman el seno familiar determina la utilidad total de la familia (ecuación a):

$$
\begin{gathered}
U(0)=\int_{0}^{\infty} u\left(c(t) e^{-\rho t} d t\right. \\
Y=Y_{L}+Y_{I} \\
Y_{L}=w(1-\propto) N+r B \\
Y_{I}=s \propto N-q \propto N=(s-q) \propto N=(s-q) D \\
T=N+R \\
\frac{d B}{d t}=Y-C=w(1-\propto) N+r B+s \propto N-q \propto N-C
\end{gathered}
$$

\section{Donde:}

$u$, utilidad individual; , ingreso total familiar; , ingreso lícito; ingreso ilícito; , total de tiempo que dispone el individuo; , Tiempo destinado a las actividades lícitas e ilícitas; cantidad de actividades delictivas o ilícitas; , proporción del tiempo destinado a las actividades delictivas; , tiempo destinado al ocio (descanso); , ingreso bruto promedio generado por cada actividad ilícita; costo promedio incurrido en cada actividad ilícita; , salario de la actividad lícita; , gasto de consumo familiar; , tasa de interés; , stock de activos financieros; , tasa de descuento; , consumo individual. 


\section{Pensamiento Crítico Vol. 20. NoI}

El proceso de maximización de utilidad está sujeto a la restricción presupuestaria, es decir, al ingreso total familiar $(Y)$. Dicho ingreso es igual a la suma del ingreso neto proveniente de las actividades delictivas o ilícitas $\left(Y_{I}\right)$ y de las actividades lícitas $\left(Y_{L}\right)$; (ecuación b).

El ingreso lícito proviene de los intereses generados por el stock de activos financieros que posee el individuo $(r B)$ y del ingreso generado por el trabajo lícito efectuado (w $(1-\alpha)$ $N$ ), donde (1- $\alpha$ ) es el tiempo disponible destinado a dicha actividad lícita; (ecuación c).

El ingreso neto que genera la actividad delictiva es igual al ingreso bruto que produce dicha actividad $(s \alpha N)$ menos el costo que implica el mismo $(q \alpha N)$. La actividad delictiva no solo genera ingresos para el individuo que la comete, sino también costos. Por ello, dicho ingreso neto delictivo se puede definir como el ingreso neto unitario del delito $(s-q)$ multiplicado con la cantidad de delitos cometidos $(D)$; (Ecuación d).

El total de tiempo que dispone el delincuente $(T)$ es igual a la suma de lo dedicado al trabajo lícito e ilícito $(N)$ y al descanso u ocio $(R)$; (ecuación e).

Finalmente, dado el carácter dinámico del modelo, el cambio del stock de activos financieros en el tiempo es igual al ingreso total, deducidos el gasto en consumo de la familia $(C)$; (ecuación f).

Con la finalidad de entender de manera más sencilla la lógica del modelo dinámico formulado para un período infinito, a continuación reexpresamos dicha lógica en términos de un modelo de solo dos períodos: 1 = período presente y 2 = período futuro.

En este modelo, en forma similar que el anterior, el individuo maximiza bienestar o utilidad $(U)$, el mismo que depende del nivel de consumo familiar alcanzado en el período uno y dos $\left(C_{1}, C_{2}\right)$; (ecuación a): 


$$
\begin{gathered}
U=U\left(C_{1}, C_{2}\right) \\
B_{1}=Y_{L 1}+Y_{I 1}-C_{1} \\
C_{2}=Y_{L 2}+Y_{I 2}+(1+r) B_{1} \\
Y_{1}=Y_{L 1}+Y_{I 1} \\
Y_{2}=Y_{L 2}+Y_{I 2} \\
C_{1}+\frac{C_{2}}{(1+r)}=Y_{I 1}+\frac{Y_{I 2}}{(1+r)}+Y_{L 1}+\frac{Y_{L 2}}{(1+r)}
\end{gathered}
$$

La maximización de utilidad está sujeta a su restricción presupuestaria. En tal sentido, el stock de activos financieros (ahorro) cumulados en el período presente -que genera intereses- es igual a su ingreso total generado por la actividad lícita $\left(Y_{L I}\right)$ y la actividad ilícita $\left(Y_{I I}\right)$ deducido el gasto en consumo $\left(C_{1}\right)$; (ecuación b).

En tanto que el nivel de gasto en consumo en el período futuro o dos $\left(C_{2}\right)$ es igual a su ingreso total generado en dicho período $\left(Y_{I 2}+Y_{L 2}\right)$ sumada la renta generada por el ahorro efectuado en el primer período $\left((1+r) B_{1}\right)$, ello asumiendo que el individuo no deja herencias; (ecuación c).

Se supone que el ingreso corriente generado del período presente $\left(Y_{1}\right)$ es igual a la suma del ingreso lícito e ilícito $\left(Y_{L I}+Y_{I I}\right)$; (ecuación d). De manera similar, el ingreso corriente en el período futuro $\left(Y_{2}\right)$ es igual a la suma del ingreso lícito e ilícito producido en dicho período $\left(Y_{L 2}+Y_{I 2}\right)$; (ecuación e).

Reemplazando la ecuación (b) en (c) y operando se halla la ecuación de la restricción presupuestaria intertemporal; (ecuación f). Esta última ecuación indica que el gasto en consumo en el tiempo, está sujeto necesariamente a su ingreso intertemporal. En términos formales, el valor presente de su flujo de gasto en consumo es necesariamente igual al valor presente de su flujo de ingresos, el mismo que incluye tanto el ingreso lícito como el generado por las actividades ilícitas o delictivas. 


\section{Pensamiento Crítico Vol. 20. NoI}

La lógica algebraica expuesta también se puede exponer en términos gráficos (véase Gráfico 1).

Gráfico 1: Maximización de bienestar del delincuente

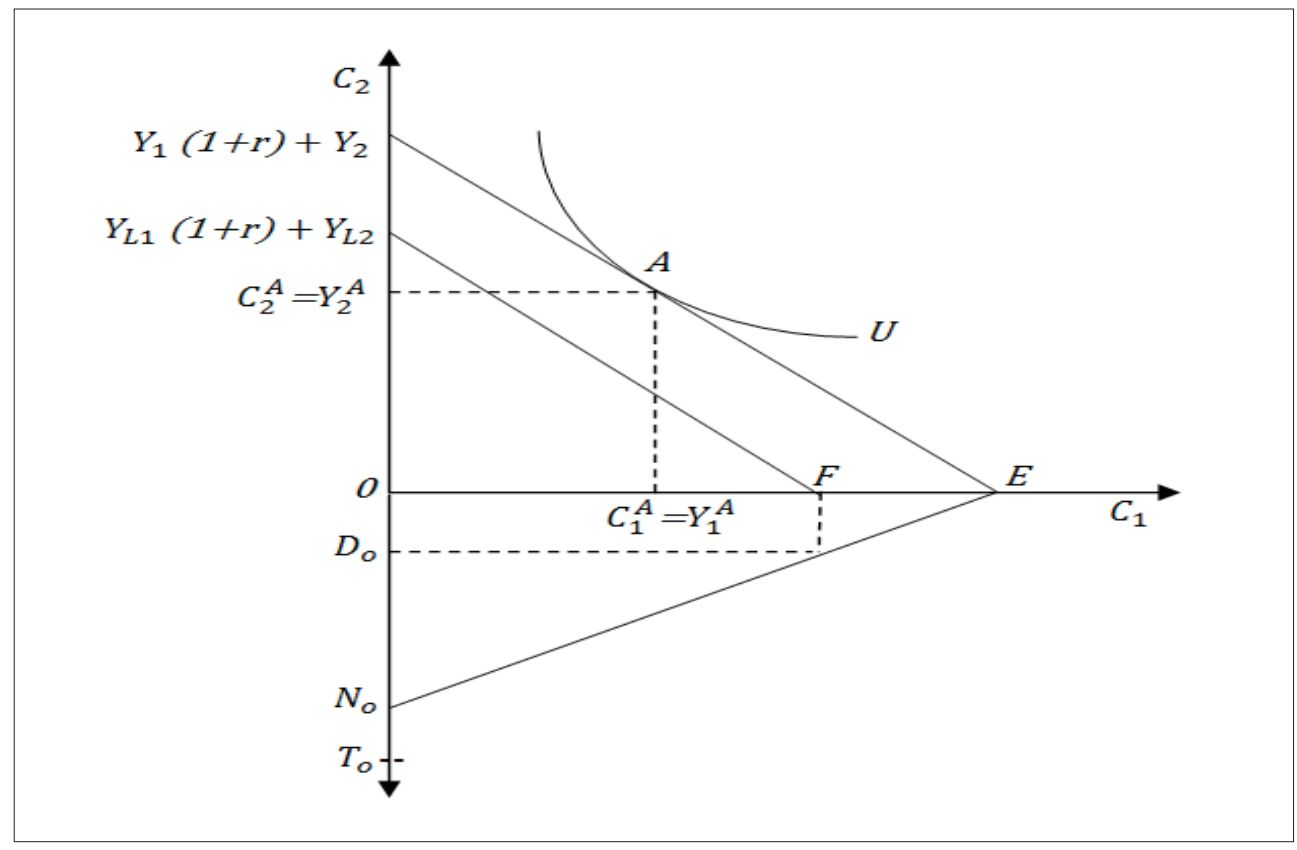

En el eje horizontal se muestra el nivel de consumo como el ingreso en términos del período presente, en tanto que en el eje vertical superior el ingreso y consumo en términos del período futuro. En el eje vertical inferior se expone el total de tiempo que dispone el individuo $\left(T_{0}\right)$, la cantidad de tiempo destinado al trabajo lícito e ilícito $\left(N_{0}\right)$ y la cantidad de delitos cometidos $\left(D_{0}\right)$.

El delincuente maximiza utilidad en el punto, donde su preferencia reflejada en la curva de indiferencia o utilidad es tangente a su recta de presupuesto. El delincuente aspira y trata de alcanzar dicho nivel de bienestar o utilidad. Su curva de restricción presupuestaria es la suma del ingreso lícito (distancia OF en términos del período presente) y el ingreso ilícito (distancia $F E$ ). 


\section{Juan León Mendoza}

El delincuente alcanza la utilidad deseada, consumiendo en el período presente, el mismo que es igual al ingreso destinado a financiar el consumo en dicho período $\left(Y_{1}^{A}\right)$. Concordante con el punto óptimo, en el período futuro su nivel de consumo es igual a $C_{2}{ }_{2}$. Por simplificación se está asumiendo que el consumo y el ingreso son iguales en ambos períodos.

Dado el ingreso lícito que posee el delincuente igual a la distancia, éste tratará de generar un ingreso mediante la actividad delictiva igual a la distancia , y así alcanzar el nivel de bienestar deseado. Para ello, el delincuente cometerá una cantidad de delitos (asaltos, robos) igual a $D_{0}$.

El modelo teórico formulado puede ser utilizado para identificar la manera en que reacciona el delincuente ante ciertos cambios o hechos. Por ejemplo, a modo de ilustración, podemos simular la respuesta del delincuente ante la caída en su nivel de ingreso lícito (distancia $F E$ ).

En el gráfico 2, la disminución en el nivel de ingreso lícito o la caída del individuo en una situación de pobreza monetaria, se traduce en un desplazamiento de la curva de presupuesto del ingreso lícito de $O F$ a $O G$.

Ante ese hecho, el delincuente que desea mantener el nivel de bienestar alcanzado en un punto A como tratará de generar el ingreso necesario mediante actividades delictivas en una distancia igual a $G E$. Si inicialmente su ingreso ilícito generado era igual a la distancia $F E$, ahora, por la disminución del ingreso lícito, necesita generar un mayor ingreso de manera delictiva igual a $G E$, por lo que cometerá más actos delictivos. Por ello, el nivel de la actividad delictiva sube de $D_{0}$ a $D_{l}$.

El modelo muestra con mucha claridad que la actividad delictiva (robos y asaltos) tiende a producirse cuando el individuo experimenta una caída en su nivel de ingreso lícito. Cuando el individuo es arrojado hacia la pobreza monetaria o cuando cae su ingreso monetario lícito, éste trata de contrarrestarlo o recompensarlo a través de una mayor actividad delictiva, de modo que intenta mantener constante el nivel de bienestar inicialmente alcanzado o deseado (máxima utilidad). 


\section{Pensamiento Crítico Vol. 20. No I}

Gráfico 2: Respuesta del delincuente ante la caída en su ingreso lícito

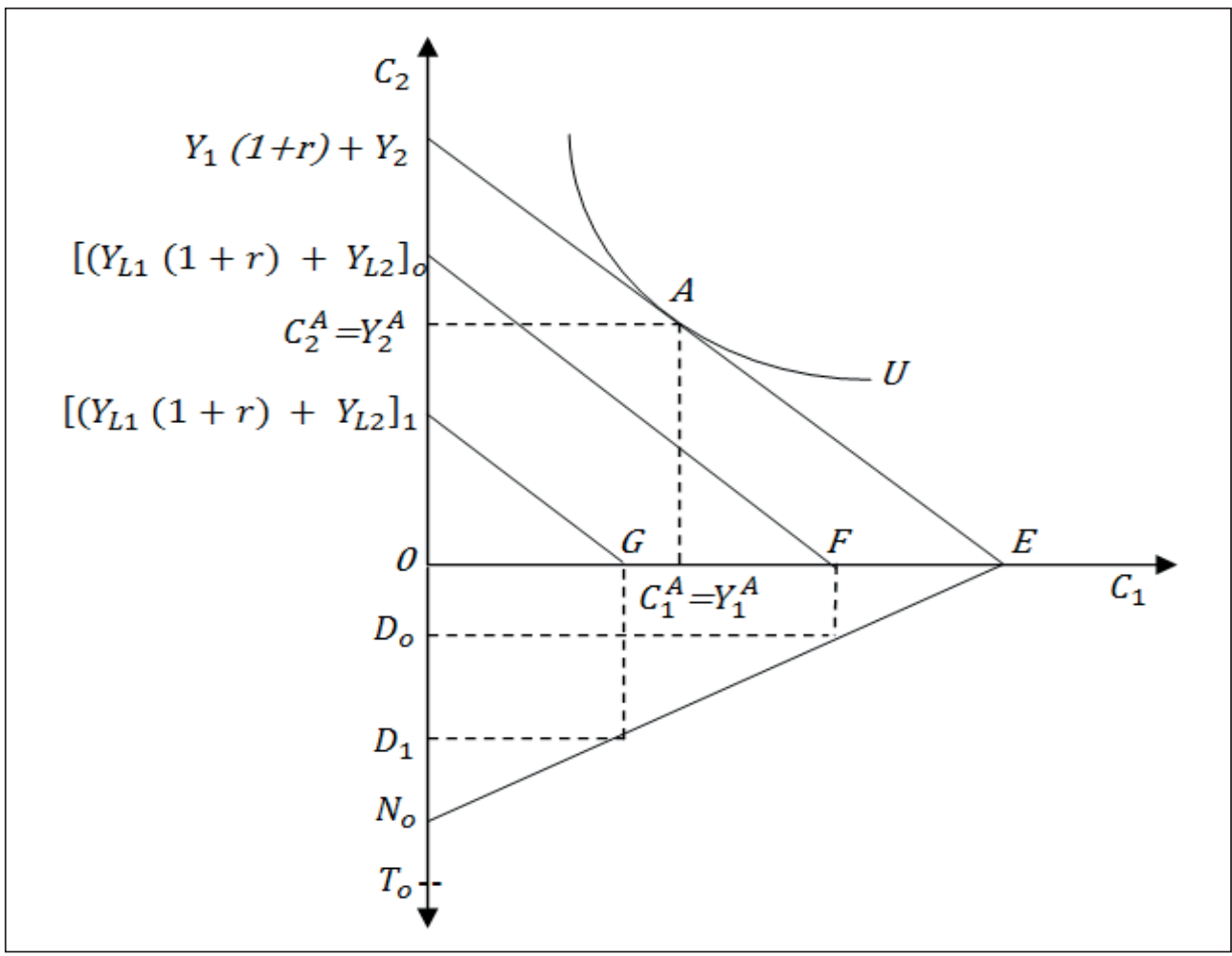

En resumen, según el modelo teórico formulado, las acciones de delito contra el patrimonio (robo y asalto) se producen, entre otros, como consecuencia de una caída en el nivel de ingreso lícito del individuo o cuando éste es arrojado a situaciones de pobreza monetaria.

\section{Aspectos metodológicos}

Teniendo en cuenta lo expuesto en el marco teórico y la lógica del modelo microeconómico formulado, efectuaremos la regresión econométrica correspondiente considerando las siguientes variables: 


\section{Juan León Mendoza}

Variables dependiente: $\mathrm{Y}=$ tasa de delitos contra el patrimonio (PATRIMONIO).

Variables explicativas: $\mathrm{X} 1=$ tasa de delito contra el patrimonio con un rezago (PATRIMONIO1), $\mathrm{X} 2$ = Tasa de consumo de droga (COMDROGA), $\mathrm{X} 3=$ Densidad Poblacional (DENSPOB), $\mathrm{X} 4=$ Desigualdad en la distribución de ingresos (DESIGUALD), X5 = Nivel de educación (EDUCACIÓN), X6 = El ingreso monetario (INGRESO), $\mathrm{X} 7$ = Tasa de población joven (POBJOVEN), $\mathrm{X} 8=$ Tasa de pobreza (POBREZA), X9 = Dotación policial (POLICIAHAB), $\mathrm{X} 10=$ Dotación de miembros de serenazgo (SERENHAB).

La tasa de delitos contra el patrimonio se halla dividiendo el número de delitos entre el número de habitantes. La tasa de consumo de droga se aproxima mediante la magnitud de la microcomercialización de la droga dividido entre la población. La data de la densidad poblacional se elabora dividiendo el número de la población entre la extensión geográfica del distrito correspondiente (población por kilómetro cuadrado). La desigualdad en la distribución de ingresos se aproxima con el coeficiente Gini. El nivel de educación se representa mediante la cantidad promedio de años estudiados por la población. El ingreso monetario se aproxima por el ingreso familiar percápita. La tasa de población joven se aproxima mediante el porcentaje de jóvenes comprendidos entre los 15 a 24 años. La tasa de pobreza se calcula como el porcentaje de la población en situación de pobreza. La dotación policial se cuantifica como el número de policías por cada 1,000 habitantes. La dotación de miembros de serenazgo se calcula como el número de miembros de serenazgo por cada 1,000 habitantes.

La información trabajada es de corte transversal y a nivel distrital, por lo que comprende a los 49 distritos conformantes de Lima Metropolitana y Callao. La información corresponde al promedio de los años 2011- 2012. Las variables regresionadas estarán expresadas en logaritmos, de modo que ello permitirá estimar directamente la elasticidad correspondiente. 


\section{Pensamiento Crítico Vol. 20. NoI}

\section{Determinantes de la generación de los delitos contra el patrimonio}

En principio, la visualización gráfica de la distribución de las observaciones del delito contra el patrimonio en los 49 distritos de Lima Metropolitana y Callao, indica que existen dos distritos con una observación relativamente muy dispersa (véase gráfico 3). Ellos son los distritos de Lurín (observación 19) y de Miraflores (observación 22). Por ello, en la regresión consideraremos dos variables dummy relacionadas a estas observaciones (D1 y D2).

Gráfico 3. Lima Metropolitana y Callao: Observación del nivel de delitos contra el patrimonio (en logaritmos)

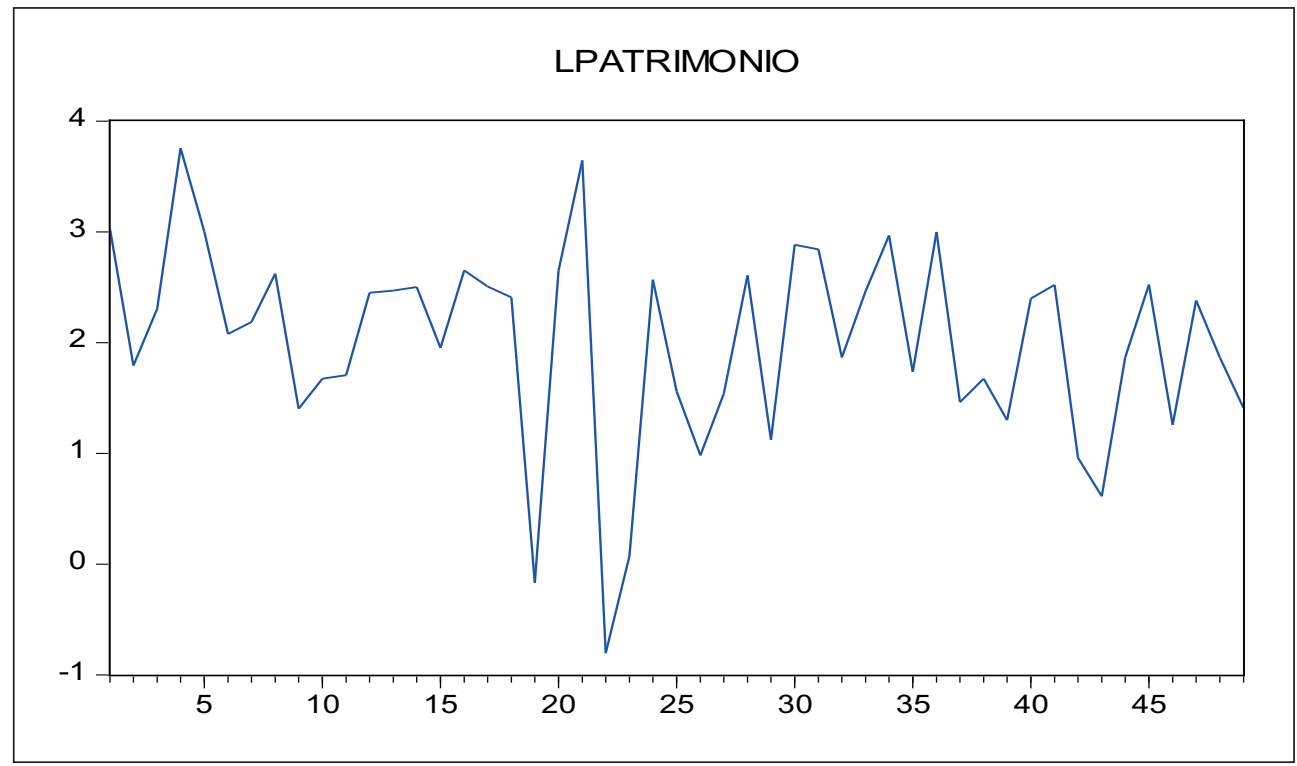

El cuadro 1 muestra el resultado de una primera regresión efectuada entre el logaritmo de los delitos contra el patrimonio (LPATRIMONIO) con respecto a las variables explicativas especificadas en el capítulo de la metodología.

El resultado de la regresión econométrica inicial muestra que el problema de los delitos contra el patrimonio está relacionado positivamente con el nivel del delito contra el patrimonio registrado en períodos anteriores, el consumo de droga, la densidad 


\section{Juan León Mendoza}

poblacional, la desigualdad en la distribución de ingresos, la pobreza, el nivel de ingreso de las familias, el nivel de la educación y la dotación policial. En tanto que la relación con la dotación de servicios de serenazgo y la proporción de la población joven es en sentido inverso.

En otros términos, a nivel de los distritos de Lima Metropolitana y Callao, los delitos contra el patrimonio ocurridos en el período corriente también se explican de manera inercial por lo ocurrido en períodos anteriores: el mayor delito en el pasado o en el presente se traduce en mayores niveles de este problema en el futuro. El delito contra el patrimonio se retroalimenta período a período.

En el mismo sentido, la violencia e inseguridad distrital relacionada con los delitos contra el patrimonio son mayores en la medida en que el distrito muestre mayores niveles de consumo de drogas, de densidad poblacional y un mayor grado de desigualdad en la distribución de ingresos.

La relación, en sentido directo, entre los delitos contra el patrimonio, con la educación, el ingreso y la dotación policial es de signo no esperado. Tampoco lo es la relación inversa con la proporción de la población joven.

La relación inversa entre los delitos contra el patrimonio y la dotación del servicio de serenazgo podría indicar que este servicio es relativamente eficaz: a mayor dotación de personal de serenazgo distrital, menor tasa de delitos contra el patrimonio.

Dada la presencia de problemas de colinealidad y heteroscedasticidad, procedemos a afinar el modelo econométrico utilizando la técnica de la matriz de correlación de variables explicativas y el test de variables redundantes.

\section{Cuadro 1: Resultado de la regresión inicial}

Dependent Variable: LPATRIMONIO

Method: Least Squares

Date: 03/13/15 Time: 19:29

Sample (adjusted): 249

Included observations: 46 after adjustments 


\section{Pensamiento Crítico Vol. 20. NI}

\begin{tabular}{lllll} 
Variable & Coefficient & Std. Error & t-Statistic & Prob. \\
\hline LPATRIMONIO1 & 0.117092 & 0.102720 & 1.139913 & 0.2625 \\
LCOMDROGA & 0.099685 & 0.126999 & 0.784932 & 0.4381 \\
LDENSPOB & 0.121639 & 0.076851 & 1.582788 & 0.1230 \\
LDESIGUALD & 4.442458 & 1.217981 & 3.647397 & 0.0009 \\
LEDUCACION & 0.533034 & 2.162334 & 0.246509 & 0.8068 \\
LINGRESO & 0.204097 & 1.008218 & 0.202433 & 0.8408 \\
LPOBJOVEN & -1.796252 & 1.000568 & -1.795232 & 0.0818 \\
LPOBREZA & 0.053253 & 0.183610 & 0.290033 & 0.7736 \\
LPOLICIAHAB & 0.285103 & 0.248833 & 1.145762 & 0.2601 \\
LSERENHAB & -0.170705 & 0.146444 & -1.165673 & 0.2521 \\
D1 & 1.641724 & 0.593763 & 2.764950 & 0.0092 \\
D2 & 3.422185 & 0.622504 & 5.497448 & 0.0000 \\
C & -5.746179 & 8.427797 & -0.681813 & 0.5001 \\
\hline R-squared & 0.775902 & Mean dependent var & 2.005072 \\
Adjusted R-squared & 0.694412 & S.D. dependent var & 0.916781 \\
S.E. of regression & 0.506797 & Akaike info criterion & 1.711671 \\
Sum squared resid & 8.475828 & Schwarz criterion & 2.228461 \\
Log likelihood & -26.36844 & Hannan-Quinn criter. & 1.905264 \\
F-statistic & 9.521405 & Durbin-Watson stat & 2.046780 \\
Prob(F-statistic) & 0.000000 & \multicolumn{5}{l}{} & \\
\hline & & \multicolumn{3}{l}{}
\end{tabular}

A su vez dejando a un lado las variables explicativas estadísticamente no significativas encontradas en las sucesivas regresiones efectuadas, se regresiona un modelo final, cuyo resultado se expone en el cuadro 2.

La regresión final indica que la variable consumo de droga es estadísticamente significativa al $10 \%$ de error, la densidad poblacional y la desigualdad en la distribución de ingresos al $1 \%$ de error, y la pobreza al $5 \%$ de error. A su vez se observa que los delitos contra el patrimonio están relacionados, en sentido directo, con el consumo de droga, la densidad poblacional y la desigualdad en la distribución de ingresos, y en sentido inverso, con el nivel de la pobreza. 
Un $\mathrm{R}$ al cuadrado de 0.709 indica que el $70.9 \%$ de la ocurrencia de los delitos contra el patrimonio en Lima Metropolitana y Callao son explicadas por las cuatro variables independientes ya señaladas o especificadas.

Un F estadístico de 16.25 señala con claridad que las variables explicativas del modelo final son, en conjunto, estadísticamente significativas.

Cuadro 2: Resultado de la regresión final

Dependent Variable: LPATRIMONIO

Method: Least Squares

Date: 03/13/15 Time: 22:06

Sample: 149

Included observations: 47

\begin{tabular}{lllll}
\hline Variable & Coefficient & Std. Error & t-Statistic & Prob. \\
\hline LCOMDROGA & 0.204010 & 0.102617 & 1.988073 & 0.0537 \\
LDENSPOB & 0.174368 & 0.047912 & 3.639367 & 0.0008 \\
LDESIGUALD & 4.228701 & 1.012207 & 4.177706 & 0.0002 \\
LPOBREZA & -0.146639 & 0.067545 & -2.170975 & 0.0359 \\
D1 & 1.888911 & 0.562228 & 3.359690 & 0.0017 \\
D2 & 3.097013 & 0.569028 & 5.442641 & 0.0000 \\
C & 1.150480 & 1.523858 & 0.754978 & 0.4547 \\
\hline R-squared & 0.709092 & Mean dependent var & 2.026789 \\
Adjusted R-squared & 0.665456 & S.D. dependent var & 0.918903 \\
S.E. of regression & 0.531492 & Akaike info criterion & 1.710345 \\
Sum squared resid & 11.29933 & Schwarz criterion & 1.985899 \\
Log likelihood & -33.19312 & Hannan-Quinn criter. & 1.814038 \\
F-statistic & 16.25010 & Durbin-Watson stat & 1.754036 \\
Prob(F-statistic) & 0.000000 & & \\
\hline
\end{tabular}

El estadístico Durbin-Watson muestra que no hay problemas de autocorrelación debido a que su valor está cerca a 2. 


\section{Pensamiento Crítico Vol. 20. NoI}

Tampoco existe el problema de la heteroscedasticidad, de modo que la distribución de los errores tiende a ser normal: el indicador Jarque-Bera hallado es de 2.15, el mismo que es mayor a 0.05 .

El gráfico 4 muestra que la bondad de ajuste es muy buena. A su vez, en la parte inferior del citado gráfico se visualiza que los residuos (la diferencia entre la data observada y la estimada) no presenta un patrón de comportamiento, es errático, por lo que no existe el problema de autocorrelación de los residuos.

De la elasticidad estimada del delito contra el patrimonio con las variables explicativas estadísticamente significativas, la elasticidad de la distribución de ingresos es la más alta.

La elasticidad del delito contra el patrimonio con el grado de desigualdad en la distribución de ingresos es de 4.23, lo que indica que -asumiendo constante a las demás variables explicativas- por cada $1 \%$ de aumento en la desigualdad de la distribución de ingresos entre cada uno de los distritos de Lima Metropolitana y Callao, el nivel del delito contra el patrimonio distrital se incrementa (o produce) en un $4.23 \%$. En otros términos, este resultado indica que los distritos donde se registre un mayor grado de desigualdad en la distribución se genera mayores delitos contra el patrimonio; es decir, en la medida en que en un distrito exista una pequeña proporción de la población con altos niveles de ingresos y una mayoritaria proporción con bajos niveles de ingresos, se producirán mayores tasas de asaltos, robos, hurtos y otros tipos delitos contra el patrimonio.

La elasticidad del delito contra el patrimonio respecto al consumo de droga es de 0.20 . Ello indica que, por cada $1 \%$ de mayor consumo de drogas a nivel de distritos, se genera un $0.20 \%$ de delitos contra el patrimonio. En otros términos, se tiende a generar mayores tasas de delitos contra el patrimonio en la medida en que sea mayor el nivel de consumo distrital de la droga: distritos que tengan una mayor cantidad relativa de consumidores de droga presentarán mayores niveles de asaltos, robos y hurtos.

La elasticidad de los delitos contra el patrimonio y la densidad poblacional es de 0.17 . Entonces, por cada $1 \%$ de mayor densidad poblacional de un distrito se genera un $0.17 \%$ más de delitos contra el patrimonio. Este resultado econométrico es esperable debido a que la mayor densidad poblacional consiste en una mayor cantidad de 


\section{Juan León Mendoza}

Gráfico 4. La bondad de ajuste

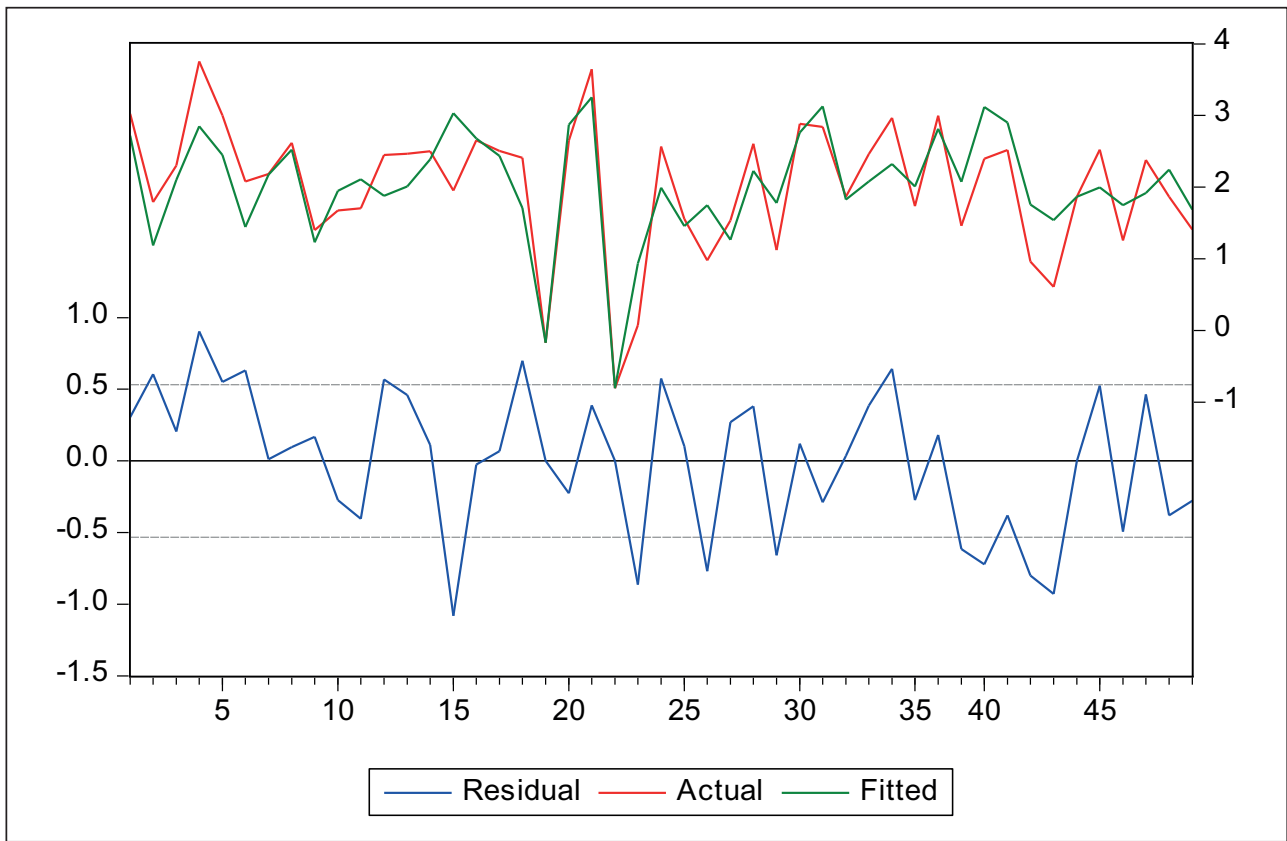

habitantes por kilómetro cuadrado. En zonas urbanas con viviendas predominantemente horizontales (con escasas edificaciones de muchos pisos), la mayor densidad poblacional significa hacinamiento y una población de bajos niveles de ingresos, por lo que no sorprende observar mayores niveles de delito contra el patrimonio.

La elasticidad de signo negativo de - 0.15 entre los delitos contra el patrimonio y la pobreza, estaría indicando que a menor nivel de pobreza del distrito se cometen una mayor cantidad de hurtos y robos. En otros términos, por cada incremento en la pobreza distrital en $1 \%$, los delitos contra el patrimonio disminuyen en $0.15 \%$.

La explicación de esta relación inversa podría estar en el hecho de que las personas que cometen los delitos de hurto y robo, si bien tienden a residir en distritos de mayor densidad poblacional y consumo de drogas (de mayores niveles de pobreza), se trasladan a los distritos de menor nivel de pobreza o con población de mayor holgura económica 


\section{Pensamiento Crítico Vol. 20. NoI}

para cometer dichos actos delictivos. Es decir, en la medida en que los distritos de menor nivel de pobreza cobijan una población promedio que posee mayores niveles de patrimonio, coadyuva a que las personas que usualmente residen en distritos con mayor pobreza, prefieran cometer sus actos delictivos en zonas de una mayor dotación de bienes o activos: para el que roba y asalta, la posibilidad de que dicho acto delictivo sea más rentable es mayor en distritos con una dotación de familias de mayor patrimonio que de menor patrimonio.

Guardando las distancias correspondientes, el que la regresión econométrica muestre que en los distritos menos pobres de Lima Metropolitana y Callao se registren mayores niveles de violencia e inseguridad relacionados a los delitos contra el patrimonio, guarda cierta similitud con lo encontrado por Reyna y Toche (1999) para el Perú. Según los citados autores, los departamentos con mayores problemas de inseguridad son aquellos donde hay menores niveles de pobreza.

\section{Referencias bibliográficas}

Becker, Gary (1968). "Crime and Punishment: An Economic Approach". Journal of Political Economy, No 76. Disponible en: http://www.jstor.org/discover/10.2307/183 0482? uid $=2 \&$ uid $=4 \&$ sid $=21101712933337$.

BID (1998). Violencia en América Latina y el Caribe: un marco de referencia para la acción. Departamento de Desarrollo Sostenible, Washington

Brender, Natalie (2012). Investigando el dilema urbano: urbanización, pobreza y violencia. Centro Internacional de Investigaciones para el Desarrollo, Toronto. Disponible en: http://www.idrc.ca/EN/Programs/Social_and_Economic_Policy/ Governance_Security_and_Justice/Documents/Researching-the-Urban-DilemmaBaseline-summary_sp.pdf

Briceño-León, Roberto (2002). "La nueva violencia urbana de América Latina". Sociologias, $N^{\circ} 8$, Porto Alegre.

Briceño-León, Roberto (2007). "Violencia urbana en América Latina: un modelo sociológico de explicación". Espacio Abierto $N^{\circ} 3$; Maracaibo, Venezuela. 


\section{Juan León Mendoza}

Disponible en: http://www2.scielo.org.ve/scielo.php?script=sci_arttext\&pid=S1315$00062007000300007 \& \mathrm{nrm}=$ iso

Davis, Jhon (1987). "The Politics of Violence. A Reassessment". Violence, aggression, Terrorism, $\mathrm{N}^{\circ} 2$. University of California, California.

Del Olmo, Rosa (2000). "Ciudades duras y violencia urbana". Nueva Sociedad, № 167. Caracas. http://www.busde.paho.org/bvsacd/cd26/foro.pdf.

Fajnzylber, P.; Lederman, D. y Loayza N.(1998). "Determinants of crime rates in Latin America and the world". World Bank Latin American and Caribbean Studies, Washington.

Hasan, Amed (2010). Can urban density be made to work foreveryone? Exploring options for Karachi's low- andlower-middle-class settlements. Environment and Urbanization, $\mathrm{N}^{\circ}$ 22. Citado por Brender (2012)

Instituto Nacional de Estadística e Informática (2013). Compendio Estadístico 2013, Lima. Disponible en: http://www.ine.cl/canales/menu/publicaciones/calendario_de_ publicaciones/pdf/COMPENDIO_2013.pdf

Jutersonke, O., R. Muggah y D. Rodgers (2009). Gangs, Urban Violence and security Interventions in Central America. Security Dialogue, $\mathrm{N}^{\circ}$ 40. Disponible en: http:// eprints.lse.ac.uk/28428/1/Gangs,_urban_violence_and_security_interventions_ (LSERO_version).doc.pdf.

Lima Cómo Vamos (2015). Encuesta Lima Cómo Vamos 2014, Lima. Disponible en:http://www.limacomovamos.org/cm/wp-content/uploads/2015/01/ EncuestaLimaComoVamos2014.pdf

Martine, G. y McGranahan, G. (2010). "Brazil's Early Urban Transition: What Can it Teach Urbanizing Countries?”. Urbanization and Emerging Population Issues, $N^{\circ} 4$. IIED, UNFPA, Londres. 


\section{Pensamiento Crítico Vol. 20. Nº}

Mascaro, P., García, S., Munares, O. (2012). "Violencia basada en género en zonas urbanas y urbano-marginales de Lima y Callao, 2007 - 2010". Anales de la facultad de Medicina, UNMSM. Lima.

Moulian, Tomás (1997). Chile actual: anatomía de un mito. LOM-ARCIS, Santiago de Chile. http://es.scribd.com/doc/58317165/Moulian-Tomas-Chile-actual-Anatomiade-un-mito-1997.

Reyna, C. y Eduardo T. (1999), "La inseguridad en el Perú", Serie Políticas Sociales, No29. Cepal, Santiago de Chile.

Stewart, Frances (2008). Horizontal Inequalities and Conflict. Understanding Group conflict in Multiethnic Societies. Basingstroke: Palgrave Macmillan. New York. Disponible en: http:/www.palgrave.com/products/title.aspx?pid=277729

Townsend, Dorn (2009). No Other Life: Gangs, Guns, and Governance in Trinidad and Tobago. SAS Working Paper, $\mathrm{N}^{\circ}$ 8. Disponible en: http://mercury.ethz.ch/ serviceengine/Files/ISN/117610/ipublicationdocument_singledocument/2ec42457cc3d-41dc-aded-b99979df5eff/en/WP8-Gangs-Guns-Governance-TrinidadTobago-2009.pdf

UNODC (2008).Handbook on planning action for Crime Prevention in Southern Africa and the Caribbean Region. Viena.

Vanderschueren, Franz (1994). "La violencia urbana, los pobres de la ciudad y la justicia”. En: Concha Eastman A, Carrión F, Cobo G, eds. Ciudad y violencias en América Latina. Programa de Gestión Urbana, Quito.

Winton, Alisa (2004). "Urban violence: a guide to the literature". Environment and Urbanization, No 16. Citado por Brender (2012). 